Karl-Dieter Opp $\cdot$ Reinhard Wippler (Hrsg.)

\title{
Empirischer Theorienvergleich
}

Erklärungen sozialen Verbaltens

in Problemsituationen

$\infty$

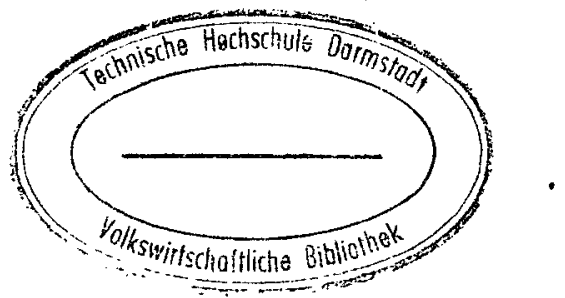

Westdeutscher Verlag 


\section{INHALT}

\section{VORWORT}

I. THEORETISCHER PLURALISMUS UND EMPIRISCHE FORSCHUNG

Karl-Dieter Opp und Reinhard Wippler

1. Gründe für die Anwendung von Theorien in der empirischen

Forschung

2. Die Auswahl der anzuwendenden Theorien

3. Die Idee des theoretischen Pluralismus: die Konfrontierung von Theorien als Aufgabe für die Sozialwissenschaften

4. Der Forschungsverbund "Vergleichende Theorientestung" 11

5. Der weitere Aufbau dieses Bandes

II. NUTZENTHEORIE UND THEORIE MENTALER INKONGRUENZEN:

DIE AUSGEWÄHLTEN INDIVIDUALTHEORIEN

Karl-Dieter Opp, Frits Tazelaar und Reinhard Wippler

1. Die Nutzentheorie

2. Die Theorie mentaler Inkongruenzen

3. Das Modell der Theorie mentaler Inkongruenzen

4. Gemeinsamkeiten und Unterschiede

III. EINE STRUKTURELLE VERSION DER THEORIE DER STATUSINKONSISTENZ

\section{Ulf Wuggenig}

1. Das Problem der divergierenden Theorieversionen

2. Die Theorieversion von Galtung $\quad 40$

2.1. Der systemtheoretische Rahmen $\quad 40$

2.2. Status, Statusinkonsistenz, Statusdiskordanz 41

2.3. Geltungsbereich und motivationale Grundlagen der Theorie 46

2.4. Makro- und Mikroeffekte von Statusinkonsistenz 49

2.5. Die Inkongruenztheorie 55

2.6. Die Inkonsistenztheorie 59

IV. DER ÜBERGANG VON HOCHSCHÜLERN VON DER UNIVERSITÄT INS BESCHÄFTIGUNGSSYSTEM

Ein Vergleich der empirischen Bewährung der Nutzentheorie und der Theorie mentaler Inkongruenzen

Elmar Lange

1. Problemstellung 71

2. Methodischer Ansatz der Untersuchung 71

2.1. Rahmenbedingungen des Theorienvergleichs 71

2.2. Forschungsdesign und Erhebungsverfahren 72 
3. Die empirische Bewährung der Nutzentheorie (NT) 74

3.1. Die problemspezifische Fassung der Nutzentheorie 75

3.1.1. Der zu erklärende Sachverhalt $\quad 75$

3.1.2.Der Erklärungsansatz $\quad 75$

$\begin{array}{ll}\text { 3.1.3.Zur Operationalisierung } & 76\end{array}$

3.2. Die Überprüfung der NT 77

3.2.1. Prüfmodelle und Häufigkeitsverteilungen der Modell-
variablen

3.2.2. Bewährung der Nutzentheorie als Dispositionstheorie 81

3.2.3. Bewährung der Nutzentheorie als Verhaltenstheorie 82

3.3. Diskussion der Ergebnisse 83

4. Die empirische Bewährung der Theorie mentaler Inkongruenzen (TMI) 85

4.1. Die problemspezifische Fassung der TMI 86

4.1.1.Die zu erklärenden Sachverhalte. $\quad 86$

4.1.2.Der Erklärungsansatz $\quad 87$

4.2. Die Überprüfung der TMI 89

4.2.1.Die Prüfmodelle und Modellvariablen 89

4.2.2. Bewährung der TMI als Dispositionstheorie $\quad 91$

4.2.3. Bewährung der TMl als Verhaltenstheorie 92

4.2.4. Bewährung der TMI in ihren Voraussagen zur Standardänderung, Realitätsleugnung und dem Auftreten von Streß 94

4.2.5. Auf dem Weg zu einfacheren Modellen der TMI 97

4.3. Diskussion der Ergebnisse 101

5. Vergleich der empirischen Bewährung der NT und der TMI 103

V. PROTEST GEGEN ATOMKRAFTWERKE

Die Erklärung von Protest durch die Nutzentheorie und die Theorie mentaler Inkongruenzen

109

Wolfgang Roehl

1. Einleitung

2. Protestintention als Erkärungsgegenstand 110

3. Erklärung von Protestintentionen mit der mentalen Inkongruenztheorie 111

3.1. Die primäre Inkongruenz 113

3.2. Sekundäre Inkongruenzen 116

3.2.1. Universelle sekundäre Inkongruenzen 118

3.2.2.Problemspezifische sekundäre Inkongruenzen 121

3.3. Perzipierte soziale Unterstützung 125

3.4. Zusammenfassung 126

$\begin{array}{ll}\text { 4. Die Nutzentheorie } & 127\end{array}$

4.1. Politische Konsequenzen von Protest 128

4.2. Externe Sanktionen 130

4.3. Intrinsische Belohnungen 132

4.4. Zusammentassung 133

5. Vergleich der Ergebnisse 134

$\begin{array}{ll}\text { Anhang } & 139\end{array}$ 
VI. BILDUNGSKAPITAL UND BERUFLICHE POSITION

Stre $B$ und Intrapunitivität als Reaktionen auf Beschäftigungsprobleme

Uwe Engel und Ulf Wuggenig

1. Das Forschungsproblem

2. Die Operationalisierung von Statusinkonsistenz und primärer Inkongruenz

2.1. Statusinkonsistenz (SI) 149

2.2. Mentale Inkongruenzen 150

3. Das Explanandum: Streß 152

4. Datenanalyse und Testkriterien 157

4.1. Das Problem impliziter Annahmen 157

4.2. Modellbildung 159

4.3. Strategie und Kriterien des vergleichenden Theorientests 161

5. Der Test der Statusinkonsistenz-Theorie 162

6. Operationalisierung und Test der Theorie der mentalen $\begin{array}{ll}\text { Inkongruenzen (TMI) } & 173\end{array}$

6.1. Die konditionalen Variablen der TMI 173

6.2. Die problemspezifischen Operationalisierungen der konditionalen Variablen der TMI

6.3. Ergebnisse des Tests der TMI • 182

7. Vergleich der Bewährung der beiden Theorien 185

VII. DIE VERARBEITUNG LANGFRISTIGER ARBEITSLOSIGKEIT 191

Frits Tazelaar

1. Einleitung

2. Vorhersagen der Statusinkonsistenztheorie 192

2.1. Statusinkonsistenz: problemspezifische Fassung 193

2.2. Reduktion der Statusinkonsistenz und strukturelle
Einschränkungen

2.3. Statusinkonsistenz: Prüfungsergebnisse 205

3. Vorhersagen der Theorie mentaler Inkongruenzen 213

3.1. Mentale Inkongruenz: problemspezifische Fassung 214

3.2. Mentale Inkongruenz: Prüfungsergebnisse 218

4. Vergleich beider Theorien hinsichtlich ihrer Erklärungsleistung 222

5. Schlußbemerkungen 226

VIII RESÜMEE: PROBLEME UND ERTRAG EINES EMPIRISCHEN THEORIENVERGLEICHS

Karl-Dieter Opp und Reinhard Wippler

1. Einige Probleme eines empirischen Theorienvergleichs 229

2. Zum Ertrag eines empirischen Theorienvergleichs 231 